\title{
Separating Response-Execution Bias from Decision Bias: Arguments for an Additional Parameter in Ratcliff's Diffusion Model
}

\author{
Andreas Voss ${ }^{1}$, Jochen $\operatorname{Voss}^{2}, \&$ Karl Christoph Klauer ${ }^{1}$ \\ ${ }^{1}$ Albert-Ludwigs-Universität Freiburg, Freiburg, Germany \\ ${ }^{2}$ University of Leeds, Leeds, UK
}

\begin{abstract}
Diffusion-model data analysis permits disentangling different processes underlying the effects of experimental manipulations. Estimates can be provided for the speed of information accumulation, for the amount of information used to draw conclusions, and for a decision bias. One parameter describes the duration of non-decisional processes including the duration of motor-response execution. In the standard diffusion model, it is implicitly assumed that both responses are executed with the same speed. In some applications of the diffusion model, this assumption will be violated. This will lead to a bias of parameter estimates. Consequently, we suggest accounting explicitly for differences in the speed of response execution for both responses. Results from a simulation study illustrate that parameter estimates from the standard model are biased if the speed of response execution differs between responses. A second simulation study shows that large trial numbers $(N>1000)$ are needed to detect whether differences in response-execution times are based on different execution times.
\end{abstract}

KEYWORDS: Response Tendency; Response-execution bias; Decision Bias; Diffusion Model; fast-dm

corresponding author:

Dr. Andreas Voss

Albert-Ludwigs-Universität Freiburg

Social Psychology and Methodology

79085 Freiburg

Germany 


\section{Separating Response-Execution Bias from Decision Bias: Arguments for an Additional Parameter in Ratcliff's Diffusion Model}

In this paper, we consider the diffusion model for fast binary decisions (Ratcliff, 1978). The model maps cognitive processes on different model parameters. Most important are the speed of information intake (drift rate) and the amount of information that is required to come to a decision (threshold). The model also captures biases in decisions. Such decision biases are mapped on the starting point of the diffusion process. Biases in starting point are often referred to as response biases (drawing on the analogous concept from Signal Detection Theory; MacMillan \& Creelman, 1991), reflecting that one response is preferred over the other response. However, we consider the label "response bias" to be misleading, because the starting point influences the decision process rather than response execution. Consequently, we denote biases based on the starting point as decision biases.

In Ratcliff's model, the total response time is composed of the decision time as described by the diffusion process, and a non-decision component that comprises processes of encoding and response execution. In previous applications of the diffusion model it has always been-implicitly-assumed that non-decision components are equal for both alternative responses. However, this assumption may be violated in many psychological tasks. Consider, for example, research on response priming (e.g., Elsner \& Hommel, 2001) or response repetition effects (e.g., Campbell \& Proctor, 1983): In these domains, the main hypotheses posit that a primed or repeated response is executed faster compared to a nonprimed or non-repeated response. In this paper, we will denote such differences in the speed of response execution between alternative responses as a response-execution bias or response tendency. This bias is theoretically independent of the decision bias discussed above, although both may often be elicited by the same tasks and may therefore occur simultaneously.

As we will show, parameters estimated with the standard diffusion model (i.e., without modelling response-execution biases) might be notably distorted if a response-execution bias is inherent in the data. To allow explicitly mapping such a bias possible, we propose to extend the diffusion model by including an additional parameter.

\section{The Diffusion Model and Predicted Response-Time Distributions}

\section{The Rationale of the Diffusion Model}

In this section, we will provide a short introduction to Ratcliff's diffusion model (e.g. Ratcliff, 1978; Ratcliff \& Rouder, 1998; Ratcliff \& Smith, 2004; Voss, Rothermund, \& Voss, 2004).

Reviews of recent developments in this area are provided by Wagenmakers (in press) and by Ratcliff and McKoon (2008).

The diffusion model is used to describe fast binary decisions. According to the model, information is accumulated continuously until evidence is sufficiently clear. The information accumulation is characterised by a constant drift and random noise. Two thresholds (for the two alternative decisions) form a corridor, in which the process runs until one of the thresholds is hit. Then the response is initiated. 
The diffusion model in its simplest form (see Figure 1) is characterised by the drift rate $(v)$, threshold separation $(a)$, starting point $(z)$, and response-time constant $\left(t_{o}\right){ }^{1}$ We will first introduce this simple model before discussing the role of additional inter-trial-variability parameters.

The average rate of information uptake is called the drift rate $(v)$. Large drift rates result in fast decisions and few errors. The distance between thresholds $(a)$ reflects the amount of information that is necessary to come to a conclusion. A large span between thresholds indicates a conservative decision style, that is, the decision is based on a large amount of information. In this case, the decision making is slow and few errors occur. A liberal decision style is represented by a small distance between thresholds.

In some cases, different amounts of information may be used for the alternative decision outcomes. This is reflected by the starting point of the diffusion process $(z)$. If the process starts above $a / 2$, comparatively less information is sufficient to come to the decision outcome associated with the upper threshold (decision A), while more information supporting $\mathrm{B}$ is needed before this decision is initiated. If the starting point is closer to the upper threshold, mean response times (RTs) of the response connected to the upper threshold will be shorter than the mean RTs of the other response. Simultaneously, the portion of responses connected to the upper threshold will increase with increasing starting point. In the simple diffusion model, any difference in mean response times between lower and upper distributions is mapped on the $z$ parameter.

The last parameter of the simple diffusion model is the response-time constant $\left(t_{0}\right)$. This parameter comprises all extra-decision processes, that is pre-decision components (e.g., stimulus encoding) and post-decision processes (e.g., response execution). Response times are modelled as the sum of decision times and response-time constant. Therefore, $t_{0}$ equally contributes to correct and error RTs.

Ratcliff's complete diffusion model allows for inter-trial variability of drift, starting point, and response-time constant. These parameters influence the shape of the predicted RT distributions, and can account for small differences in the mean RTs of correct responses and errors (e.g., Ratcliff \& Rouder, 1998): Inter-trial variability of the starting point can explain fast error responses, while inter-trial variability of the drift causes slow errors.

\section{Applications of the Diffusion Model}

The diffusion model has been applied successfully to a variety of tasks (see Spaniol, Madden, \& Voss, 2006, for a review). One main field of application is the analysis of aging effects on cognitive processes (e.g., Ratcliff, Thapar, \& McKoon, 2006, 2007). In this domain research focuses on the question whether slowing of RTs is based on slower information processing (drift), on slower response execution (RT constant), or on a more conservative decision style (threshold). One main result is that age-related slowing in cognitive tasks is in large part due to conservative threshold settings and slower motor responses, while cognitive processing is often quite robust.

Other applications aim at improving the understanding of specific cognitive tasks: For example, Klauer, Voss, Schmitz, and Teige-Mocigemba (2007) analysed data from the Implicit Association Test (IAT; Greenwald, McGhee, \& Schwartz, 1998) with a diffusionmodel analysis: Results of these analyses helped to clarify why different test blocks have different difficulties. In the same study, the inter-individual differences in parameter values were used successfully to predict explicit attitudes. This hints that the diffusion model may be used as a diagnostic tool (Schmiedek, Oberauer, Wilhelm, Süß, \& Wittmann, 2007).

1 Additionally, the diffusion process is characterised by the amount of noise. This is reflected by the diffusion constant, which operates as a scaling parameter. The diffusion constant is not estimated but can be freely chosen. 
There is a growing number of further applications of the diffusion model (see Wagenmakers, in press). The model is likely to become even more popular, due to recent publications of different software tools for diffusion-model analyses (fast-dm: Voss \& Voss, 2007; dmat: Vandekerckhove, \& Tuerlinckx, 2007, 2008; EZ-diffusion, Wagenmakers, Van der Maas, \& Grasman, 2007; Wagenmakers, van der Maas, Dolan, \& Grasman, 2008).

\section{Mapping Response Tendencies: A Second Response-Time Constant}

The diffusion model as described above might be used to detect decision biases. Consider, for example, the results of Voss, Rothermund, and Brandtstädter (2008): They showed within a motivated-perception design that participants were more liberal in the decision that an ambiguous stimulus is positive (i.e., a small amount of information is enough) and participants were more conservative in classifying a stimulus as negative (i.e., a high amount of information is used). In the diffusion model, this effect is reflected by a starting point which is close to the "positive" threshold.

The observed starting-point asymmetry in the data of Voss et al. (2008) is ultimately a consequence of shorter RTs for positive decisions (i.e., when a stimulus was classified as positive). In the diffusion model, differences in mean RTs between two alternative responses always map on the starting-point parameter $(z)$, indicating different response criteria (Ratcliff, 1985). ${ }^{2}$ However, different response criteria are not the only theoretically plausible source of differences in mean RTs between two alternative responses. Imagine, for example, a task in which stimuli have to be classified by pressing a key to the right-hand side or to the left-hand side of a keyboard, respectively. Independent of the classification rules, a right-handed person may be faster with the "right" response compared to the "left" response. If this would be mapped on the starting-point parameter, one would conclude that a more liberal criterion is associated with the corresponding decision. From a theoretical point of view, however, an effect of handedness is based on speed of response execution.

There are different psychological paradigms in which response facilitation or response inhibition are discussed as explanations of the observed effects (cf. affective priming: Fazio, Sanbonmatsu, Powell, \& Kardes, 1986; flanker task: Eriksen, Coles, Morris, \& O'Hara, $1985)^{3}$. In its standard form, the diffusion model is unable to map response tendencies and it is therefore not possible to test contrasting approaches against each other (e.g., enhanced perception vs. speeded response execution). Notice, that both approaches predict differences in latencies between responses. Therefore, we suggest applying different response-time constants for the two responses: In the extended model the RT-constant $t_{0, \text { upper }}$ is added to the decision time if the process terminates at the upper threshold, and $t_{0, \text { lower }}$ is added for all responses at the lower threshold. We will recode these parameters as $t_{0}=0.5\left(t_{0, \text { upper }}+t_{0 \text {, lower }}\right)$ and $d=t_{0, \text { lower }}-t_{0, \text { upper }}$. Hence, $t_{0}$ reflects the mean RT constant, and $d$ reflects the responseexecution bias. In the extended model, differences in mean RTs for the two responses can be either mapped by asymmetries in criteria (decision bias) or by asymmetries in responseexecution times (response-execution bias).

2 If data are analyzed in terms of correct versus erroneous responses (rather than response " $\mathrm{A}$ " vs. response "B") differences in mean RTs can be captured to a certain degree by the inter-trial variability of starting point or drift as well (Ratcliff \& Rouder, 1998). However, inter-trial variability cannot explain that-for example -response " $\mathrm{A}$ " is always faster than response "B", if this pattern holds for stimuli of type A (response "A" is correct) and of type B (response " $\mathrm{B}$ " is correct).

3 However, the response-execution bias can only explain effects in RT data and not in accuracy data. Since there are accuracy effects in affective priming, response-execution biases cannot explain the total effect. 


\section{Simulation Study 1: Recovering Parameter Values}

The aim of this study is to test whether parameter values could be recovered reliably with the extended model. For this purpose, data sets were simulated from four parameter sets, including (a) no bias in starting point or RT-constants, (b) a bias in starting point but not in RT-constants, (c) a bias in RT-constants but not in starting point and, (d) both types of bias. From these simulated data sets parameters were re-estimated using models with different restrictions.

\section{Simulating data sets}

Table 1 shows the parameter values used for the simulation of data sets. Parameter-set 1 represents a symmetric model without response-execution bias or starting-point bias. In parameter sets 2 and 4 the starting point $z$ lies above $a / 2$, indicating a decision bias in favour of response $\mathrm{A}$ (upper threshold). In parameter sets 3 and 4 a response-execution bias is simulated: Response A is executed $50 \mathrm{~ms}$ faster compared to response $\mathrm{B}$.

From each of the four parameter sets of Table 1, 100 data sets with 500 trials each (half of the trials using $v_{1}$ and half using $v_{2}$ ) were simulated using an adapted version of the construct-samples tool of fast-dm release 29 (Voss \& Voss, 2007; precision $=5.0$ ).

\section{Re-estimating parameter values}

From each of the 400 simulated data sets, parameters were re-estimated using an adaption of fast-dm-29 (precision $=4.0){ }^{4}$ For each data set parameters for four models with different restrictions were fitted: In the "fixed" model, neither decision bias nor response-execution bias were allowed ( $z=a / 2$ and $d=0)$. For the second model, only a response-execution bias was prevented $(d=0)$. The third model did not allow for a decision bias $(z=a / 2)$. Finally, the "complete" model estimated both $z$ and $d$. In summary, each of the 4 (parameter sets) $\times 100$ data sets was analyzed with 4 (parameter constraints) different estimation procedures. The different restrictions were used to test the effect of "wrong" restrictions (e.g., fixing $d=0$, when $d=0.05$ in the original data set). Additionally, it was thus possible to test whether the "true" source of RT differences between both responses could be identified.

Means and standard deviations for the parameter estimates are presented in the Figures 2 and 3. Panels A to D show results from parameter sets without biases (panel A), with a decision bias on the starting point (panel B), with a response-execution bias (panel C), and with both types of biases (panel D). The estimates for the variability parameters are presented in Table 2.

\section{Parameter Set 1: No Bias}

Panel A of Figure 2 shows the mean estimates for data from parameter set 1 . All parameters were estimated accurately by all four models. This was expected, because no restrictions conflicted with the original parameter values.

4 Inclusion of a second response time constant in fast-dm is a relatively small change and will be included in one of the next releases of the program. A problem arises if the Kolmogorov-Smirnov Statistic (i.e., the maximum vertical distance between predicted and observed CDF) is used as an optimisation criterion: In the extended model, changing a single RT constant influences only one of the two predicted RT distributions (upper or lower). Thus, when the point of maximum distance and the changed RT constant correspond to opposite sides, the KS-statistic will be unaffected by changes of one of the RT constants within a certain range and minimising the vertical distance no longer results in unique parameter values. To solve this problem the sum of the maximum vertical distances of the upper and the lower distribution was used as minimisation criterion. 


\section{Parameter Set 2: Decision Bias}

In parameter set 2, the starting point was biased towards the upper threshold. Therefore, restrictions in the Models 1 and $3(z=a / 2)$ do not allow an accurate recovering of the original values. As evident in panel B of Figure 2, an erroneous restriction of the starting point leads to a biased estimation of drift rates, that is, the low starting-point value results in an inflation of the estimates for the drift. If $z$ was restricted, but the response-execution bias parameter $d$ was estimated (Model 3), an erroneous response-execution bias of $50 \mathrm{~ms}$ was estimated. In the complete model (Model 4), the mean estimates for the starting point and responseexecution bias were close to the correct values.

\section{Parameter Set 3: Response-Execution Bias}

Parameter-set 3 represents the critical case of data sets with inherent response tendencies $(d=0.05)$. Panel $\mathrm{C}$ of Figure 3 depicts the results of the parameter estimation procedure. In the standard analysis $(d=0)$, the starting point is notably overestimated, while the estimates for the drift rate fall below the true values. The models accounting for $d$ yield much more precise results. However, if $z$ is not fixed to the correct value, then the bias is dispersed to a certain degree between $z$ and $d$, that is, results show a bias in $z$ (although there was none in the data), while the estimate for the response-execution bias falls short of the true value of $50 \mathrm{~ms}$.

\section{Parameter Set 4: Decision Bias and Response-Execution Bias}

Panel D of Figure 3 depicts the results for the data sets with decision bias and responseexecution bias. The restricted models show the compensatory effects of both bias parameters: In the model without response-execution bias $(d=0)$, the decision bias is overestimated $(\mathrm{z}>0.65)$; in the model without decision bias $(z=a / 2)$, the response-execution bias is overestimated $(d>0.05)$. Estimates are most accurate in the complete model accounting for both types of biases. However, in the complete model again the estimates for the responseexecution bias are too low.

\section{Model Fit}

The optimisation criterion of fast- $d m$, that is, the Kolmogorov-Smirnov statistic (KS) is reported here as an indicator for model fit (Voss, Rothermund, \& Voss, 2004). Separate KSvalues were computed for the two trial types $\left(v_{1}\right.$ vs. $\left.v_{2}\right)$. Table 3 shows the mean KS values across both trial types. Low values indicate a good model fit. The model without biases is taken as a baseline condition here. Accordingly, a KS value below .030 is regarded as a good model fit. As evident in Table 2, the fit-index exceeds this value in each case in which a model restriction violated original values from parameter sets.

\section{Discussion}

Study 1 was conducted to demonstrate that incorrect restrictions in the diffusion model result in notably biased parameter estimates. Specifically, this was illustrated for the case of response-execution biases, that is, for a situation in which one response is executed faster than the alternative response. Standard diffusion model analyses so far did not account for differences in speed of response execution.

A response-execution bias that is not accounted for by the model is mapped on a (congruent) bias on starting point and - simultaneously — on an (incongruent) bias on the drift (see Ratcliff \& McKoon, 2008, for a discussion of the criterion of drift rates). For example, when the response linked to the upper threshold is executed faster, and this is not mapped in the model, the starting point will be overestimated and the drift will be underestimated.

Results also evidence difficulties of estimating $d$ and $z$ at once, even in data sets with 500 trials as used in the present studies. However, the comparison of fit indices showed that 
on average models accounting for the "correct" type of bias had a slightly better fit. We investigate this issue more closely in Study 2.

\section{Simulation Study 2: Identifying Response-Execution Biases in Individual Data Sets.}

Study 1 showed that it can be challenging to get reliable estimates for $z$ and $d$ simultaneously. Including both parameters makes the model so flexible that the solution of a parameter search is not robust. Therefore, we recommend using models that either allow for starting-point asymmetries or for response-execution biases, but not for both kinds of bias. Study 2 systematically examines how many trials are needed to reliably decide which of these two models better accounts for an individual data set. For this purpose, 1000 data sets were simulated from parameter sets with starting-point asymmetries (but no response-execution bias) and from parameter sets with response-execution bias (but no bias on starting point) for four different sample sizes $(500,1000,2000$, and 5000 trials per data set). Subsequently, fitindices were compared between these two models. Model fit was assessed using the KSstatistic.

\section{Simulating Data Sets and Re-Estimating Parameter Values}

In a first step, 1000 parameter sets were generated by randomly drawing values for $a, v_{1}, v_{2}, t_{0}$, $s_{z}, s_{v}$, and $s_{t 0}$ from uniform distributions (see Table 4). From each of these parameter set, two models were derived: For the starting-point bias model, $z$ was calculated as $0.65 a$, and $d$ was set to 0 . For the response-execution model, $d$ was set to 0.05 seconds, and $z$ was set to $0.5 a$. For all parameter sets and both models, data sets with 500, 1000, 2000, and 5000 trials were simulated using a modified version of the construct-samples (precision $=4$ ) tool of fast- $\mathrm{dm}$. In each case, two stimulus types were simulated, that is $v_{l}$ and $v_{2}$, respectively, were used for half of the trials of each data set.

From each of the resulting 8000 data sets, two parameter sets were re-estimated using an adapted version of fast- $d m$ (precision $=3$ ), whereat either $z$ was fixed to 0.5 a (responseexecution model) or $d$ was fixed to 0 (starting-point bias model).

\section{Results}

Each data set was analysed regarding whether the response-execution model or starting-point bias model fitted the data better in terms of the KS-statistic. Figure 4 shows the frequency of false classifications as a function of the original model and sample size. As expected, the probability of false model selection declines with increasing number of trials. Results indicate that a response-execution bias of $d=.05$ is more often correctly classified compared to a starting-point bias of $z=0.65 a$. In the former case, ca. 900 trials are sufficient for $95 \%$ correct classifications, while in the latter case about 1750 trials are needed to achieve the same accuracy.

\section{Discussion}

Study 2 demonstrates that is possible to decide for an individual data set whether a bias in mean response times is based on differences in response execution (response-execution bias) or on differences in information accumulation (starting point bias). Because the diffusion model very flexible accounts for different shapes of RT-distributions (especially if the variability parameters are included), many trials are needed to allow for reliable separation of both models: Results of the present experiment indicate that at least 1000 trials are needed, 
even in the case of large effects (i.e., about 50ms difference between mean latencies for the two responses). To get an estimate for the number of trials required when effects are notably smaller, similar simulation studies can be repeated for other parameter sets.

\section{Model Mimicry: Locating the Effect of Wrong Parameter Specifications in the RT Distributions}

As shown above, it is difficult to separate the response-execution bias model from the starting-point bias model. That is, even if there are invalid model specifications (e.g., $d=0$ ), the "wrong" model can mimic the empiric RT distribution. In this section, we further analyze where exactly the predicted RT-distribution differs from the empiric distributions in case of wrong specifications.

For this purpose, two "non-random" data sets were simulated ${ }^{5}$ for parameter sets using the means of the parameter distributions of Study $2\left(a=1.05 ; v_{1}=2.50 ; v_{2}=-2.50 ; t_{0}=0.300\right.$; $\left.s_{z}=0.13 ; s_{v}=0.50 ; s_{t 0}=0.05\right)$ one set with $d=0.05$ and $z=0.50 a$ and one set with $d=0$ and $z=$ $0.65 a\left(N=10000\right.$ trials, half of these based on $v_{1}$ and half based on $v_{2}$, respectively).

Parameters were re-estimated using "wrong" restrictions, that is, fixing $d=0$ for the data generated based on the response-execution bias model and fixing $z=0.5 a$ for the data generated on basis of the starting-point bias model.

Distributions of the data were compared with predicted distributions from the recovered parameters. For this purpose, so-called combined cumulative distribution functions (cCDF; see Voss et al, 2004; Voss \& Voss, 2008) were used. The cCDF-graphs combine the RT distributions for the two responses by mapping the times from the lower distribution of the diffusion model on the negative half-axis. The lower distribution is mirror-imaged, that is, the tail of the distribution is depicted on the far left of the graph and the starting edge of the RT distribution appears close to the origin (Figure 5). The height of the plateau around zero indicates the total mass of the lower distribution.

Figure 5 shows the cCDFs of original parameters and of parameter estimates from the models with restriction that are incompatible with the fitted data. Despite the wrong restrictions, deviations are rather small: Especially the leading edges of the RT distributions are recovered very well. The strongest deviations are evident at the tails of the RT distributions: For the response-execution bias model, the predicted $\mathrm{cCdF}$ of stimulus 1 approaches 0 on the left-hand side and 1 on the right hand side more slowly than the $\mathrm{cCdF}$ of the original data. That is, the predicted number of slow responses exceeds the actual number. The opposite is true for stimulus 2: Here, the predicted $\mathrm{cCdF}$ approaches 0 and 1 faster than the original $\mathrm{cCdF}$, that is, the tails of the RT-distributions are underestimated. The pattern of results is reversed for the starting-point bias model: Here, the tails of distributions are underestimated for stimulus 1 and overestimated for stimulus 2 .

In summary, these graphs suggest that the tails of RT distributions might be especially indicative for these kinds of biases: To make a decision, one has to consider separately stimuli corresponding to the faster response and stimuli corresponding to the slower response: If the percentage of slow responses is overestimated for stimuli requiring the faster response and the percentage of slow responses is underestimated for stimuli requiring the alternate response, this may be taken as an indicator for an unaccounted response-execution bias. The opposite pattern of results, that is, an underestimation of slow latencies for the stimuli with fast responses and vice versa suggests that there might be a decision bias in the data.

However, we emphasise that the location of mismatch between data distributions and predicted distributions might strongly depend on the optimisation criteria: The application of

5 The data points were located on a grid of regularly spaced quantiles of the predicted distributions. 
the KS statistic leads to a nearly exact recovery of the leading edges of the RT distributions of correct responses, which comes at the cost of a poor recovery of the last quantiles of the RT distributions, and - generally — of error distributions. This might be different if, for example, the $\mathrm{Chi}^{2}$ statistic was applied.

\section{General Discussion}

In this paper, we introduced an extension to Ratcliff's (1978) diffusion model. In the standard diffusion model, the assumption is implicitly made that there is no (systematic) difference between the speed of execution of both possible responses. The model's extension aims to allow mapping differences in speed of response execution for the two different responses. With estimation procedures using the standard model (e.g., fast-dm: Voss \& Voss, 2007, 2008; dmat: Vandekerckhove \& Tuerlinckx, 2007, 2008), differences in response speed between conditions can be mapped, if the $t_{0}$ parameter is allowed to vary between conditions. However, in this case, $t_{0}$ depends on condition and not on response, that is, within each condition again the same execution time is assumed for the two alternative responses " $\mathrm{A}$ " and "B".

Simulation Study 1 investigates the effects of wrong model restrictions (especially the assumption that there is no response-execution bias) and checks whether it is possible to recover a response-execution bias accurately. Results indicate that outcomes of a diffusionmodel analysis are seriously affected if an existing response-execution bias is not incorporated in the model. Specifically, the estimate for the starting point will be biased towards the threshold linked to the faster response, while the drift will be biased in the opposite direction. At the same time, our findings demonstrate that it might be challenging to estimate both $d$ and $z$ simultaneously. One strategy to deal with this problem is the comparison of model fit between models that account either for a starting-point bias or for a response-execution bias. Unfortunately, there is no exact way to test whether the inclusion of a new parameter improves the fit significantly. However, rules-of-thumb can be generated to decide which restrictions are sensible. If the fit between models differs only a little (e.g. by less than 1 SD; see Table 3), the more parsimonious model should be chosen (if the number of parameters is equal, of course the better fitting model is chosen). If the difference in fit is large (e.g., more than $1 \mathrm{SD}$ ), the inclusion of the additional parameter seems to be justified. Of course, this heuristic is developed post-hoc and needs to be tested with broader simulation studies, including other parameter values and trial numbers.

While the heuristic explicated above allows for comparison of model fit for a group of participants, it does not help to decide for an individual data set whether the decision-bias model or the response-execution model accounts for the data better. For this purpose, in simulation Study 2 we examined how many trials were needed to ensure that the true model resulted in a better model fit with a probability of $p=.95$. Results indicated that $N=1000$ to 2000 trials were needed to reliably detect the true model, even for large asymmetries as used in the present study. Consequently, for many psychological experiments the collected amount of data will not suffice to detect a moderate response-execution bias. It might be helpful to compare fit indices at a group level, which was successful in Study 1. If data sets are even smaller $(N<500)$, the problem of discrimination between the alternate models obviously becomes even more severe.

\section{Potential Biases of Parameter Estimates from the Standard Model}

How can the biases of the parameter estimates from the standard model be explained? To answer this question one must have a look on the influence of the diffusion model's parameter on the predicted response times: First of all, it is important to note that the drift rate has 
parallel effects on predicted RTs for correct responses and errors: The larger the drift, the faster the mean response times. As long as the starting point is symmetrical $(z=0.5 a)$ and all inter-trial variabilities are zero, the predicted RTs for errors and correct responses are exactly identical. There is, however, a strong influence of the starting point on predicted RTs: If the starting point moves upward, the predicted exit times for the upper threshold decrease, and times for the lower threshold increase. To put it the other way around, if there is an empirical difference in mean RTs, the standard diffusion model will map this on the starting point.

By moving the starting point, differences in mean RTs of the upper and lower threshold can be accommodated. However, moving the starting point upward increases the percentage of predicted responses for the upper threshold. If the asymmetric starting point is an artifact, that is, if it is not based on real different threshold settings for the information accumulation, the number of predicted responses will be overestimated at the threshold towards which the starting point has moved. In the multi-dimensional parameter search this will be compensated for by an opposite effect on drift rate. In summary, if response " $\mathrm{B}$ " is delayed, the starting point will be biased towards " $A$ " and the drift will be biased towards "B".

\section{Potential Benefits of Additional Information from the Extended Model}

In the above discussion we concentrated on drawbacks for the validity of the parameter estimates (especially for $z$ and $v$ ) if only one RT constant is adopted. However, this is not the only - and perhaps not the most important - reason to use the extended diffusion model. The most fundamental benefit of the extended model is that it allows for testing new empirical hypotheses. For any theory predicting response preparation, response facilitation, response inhibition, or response conflicts, the crucial tests regard differences in the speed of execution of the alternative responses (but see Footnote 3). For this purpose, different RT-constants as implemented in the extended diffusion model are required.

Imagine, for example, the case of affective priming (Fazio et al., 1986). There is a lively debate about whether this effect is equivalent to semantic priming (i.e., the prime preactivates the target-concept via spreading activation), or whether affective priming works similar to response priming (i.e., the prime prepares a specific response). In the former case, an affectively congruent priming should facilitate target perception (i.e., increase the drift), while in the latter case, a congruent prime should decrease the RT constant of the correct response (and increase the RT constant of the alternative response). With the extended model it is possible to differentiate between these hypotheses empirically. Note, however, that response execution alone cannot explain the complete affective priming effect, if effects are not limited to RTs but are also present in errors.

One caveat concerns the interpretation of the RT constant. The RT-constants map all non-decision parts of information processing, that is, stimulus encoding as well as response execution. Therefore, it is theoretically possible that differences between the RT-constants are based on differences in encoding times. However, in most experimental designs, stimuli do not differ in complexity, and no large differences in encoding times are to be expected.

We are optimistic that the extension of the diffusion model might prove useful for analysing data from many psychological paradigms. To make this kind of analysis accessible to a broader community of researchers, the model's extension will be implemented in one of the next releases of the fast-dm software (Voss \& Voss, 2007; available from http://www.psychologie.uni-freiburg.de/Members/voss/fast-dm). 


\section{References}

Campbell, K. C. \& Proctor, R. W. (1993). Repetition effects with categorisable stimulus and response sets. Journal of Experimental Psychology: Learning, Memory, and Cognition, 19(6), 1345-1362.

Conover, W.J. (1999) Practical nonparametric statistics. New York: Wiley.

Elsner, B. \& Hommel, B. (2001). Effect anticipation and action control. Journal of Experimental Psychology: Human Perception and Performance, 27(1), 229-240.

Eriksen, C. W., Coles, M. G., Morris, L. R., \& O'Hara, W. P. (1985). An electromyographic examination of response competition. Bulletin of the Psychonomic Society, 23(3), 165-168.

Klauer, K. C., Voss, A., Schmitz, F., \& Teige-Mocigemba, S. (2007). Process components of the implicit association test: A diffusion-model analysis. Journal of Personality and Social Psychology, 93(3), 353-368.

Fazio, R. H., Sanbonmatsu, D. M., Powell, M. C., \& Kardes, F. R. (1986). On the automatic activation of attitudes. Journal of Personality and Social Psychology, 50(2), 229-238.

Grasman, R. P. P. P., Wagenmakers, E.-J., \& van der Maas, H. L. J. (2008). EZ2: An extension of the EZ diffusion model for response time and accuracy. Manuscript submitted for publication.

Greenwald, A. G., McGhee, D. E., \& Schwartz, J. L. K. (1998). Measuring individual differences in implicit cognition: The implicit association test. Journal of Personality and Social Psychology, 74(6), 1464-1480.

Macmillan, N. A. \& Creelman, C. D. (1991). Detection theory: A user's guide. New York, NY: Cambridge University Press.

Ratcliff, R. (1978). A theory of memory retrieval. Psychological Review, 85(2), 59-108.

Ratcliff, R. (1985). Theoretical interpretations of the speed and accuracy of positive and negative responses. Psychological Review, 92(2), 212-225.

Ratcliff, R., \& McKoon, G. (2008). The diffusion decision model: Theory and data for twochoice decision tasks. Neural Computation, 20(4), 873-922.

Ratcliff, R. \& Rouder, J. N. (1998). Modeling response times for two-choice decisions. Psychological Science, 9(5), 347-356.

Ratcliff, R., Thapar, A., \& McKoon, G. (2006). Aging and individual differences in rapid twochoice decisions. Psychonomic Bulletin \& Review, 13(4), 626-635.

Ratcliff, R., Thapar, A., \& McKoon, G. (2007). Application of the diffusion model to twochoice tasks for adults 75-90 years old. Psychology and Aging, 22, 56-66. 
Ratcliff, R., \& Smith, P. L. (2004). A comparison of sequential sampling models for twochoice reaction time. Psychological Review, 111(2), 333-367.

Schmiedek, F., Oberauer, K., Wilhelm, O., Süß, H.-M., \& Wittmann, W. W. (2007). Individual differences in components of reaction time distributions and their relations to working memory and intelligence. Journal of Experimental Psychology: General, $136(3), 414-429$.

Spaniol, J., Madden, D. J., \& Voss, A. (2006). A diffusion model analysis of adult age differences in episodic and semantic long-term memory retrieval. Journal of Experimental Psychology: Learning, Memory, and Cognition, 32(1), 101-117.

Vandekerckhove, J. \& Tuerlinckx, F. (2007). Fitting the Ratcliff diffusion model to experimental data. Psychonomic Bulletin \& Review, 14(6), 1011-1026.

Vandekerckhove, J. \& Tuerlinckx, F. (2008). Diffusion model analysis with MATLAB: A DMAT primer. Behavior Research Methods, 40, 61-72.

Voss, A., Rothermund, K. \& Brandtstädter, J. (2008). Interpreting ambiguous stimuli: Separating perceptual and judgmental biases. Journal of Experimental Social Psychology, 44, 1048-1056.

Voss, A., Rothermund, K., \& Voss, J. (2004). Interpreting the parameters of the diffusion model: An empirical validation. Memory \& Cognition, 32(7), 1206-1220.

Voss, A. \& Voss, J. (2007). Fast-dm: A free program for efficient diffusion model analysis. Behavior Research Methods, 39(4), 767-775.

Voss, A. \& Voss, J. (2008). A fast mumerical algorithm for the estimation of diffusion-model parameters. Journal of Mathematical Psychology, 52, 1-9.

Wagenmakers, E.-J. (in press). Methodological and empirical developments for the Ratcliff diffusion model of response times and accuraccy. European Journal of Cognitive Psychology.

Wagenmakers, E.-J., van der Maas, H. L. J., \& Grasman, R. P. P. P. (2007). An EZ-diffusion model for response time and accuracy. Psychonomic Bulletin \& Review, 14(1), 3-22.

Wagenmakers, E.-J., van der Maas, H. L. J., Dolan, C. V., \& Grasman, R. P. P. P. (2008). EZ does it! Extensions of the EZ-diffusion model. Psychonomic Bulletin \& Review, 15(6), 1229-1235. 
Andreas Voss, Institut für Psychologie, Universität Freiburg. Jochen Voss, Department of Statistics, University of Leeds. Karl Christoph Klauer, Institut für Psychologie, Universität Freiburg, Correspondence concerning this article should be addressed to Andreas Voss, Institut für Psychologie, Universität Freiburg, D-79085 Freiburg, Germany, E-Mail: voss@psychologie.uni-freiburg.de.

The research reported in this paper was supported by grant K1 614/31-1 from the Deutsche Forschungsgemeinschaft to Prof. Dr. Karl Christoph Klauer. 


\section{Tables}

Table 1. Parameter values used for the simulation of data sets (study 1). Moderate variability was assumed $\left(s_{z}=0.2 ; s_{v}=0.5 ; s_{t 0}=0.1\right)$.

\begin{tabular}{lllll}
\hline & \multicolumn{4}{c}{ Parameter Set } \\
\hline Parameter & \multicolumn{1}{c}{1} & \multicolumn{1}{c}{2} & \multicolumn{1}{c}{3} \\
\hline Threshold separation & $a=1.00$ & $a=1.00$ & $a=1.00$ & $a=1.00$ \\
Starting point & $z=0.50$ & $z=0.65$ & $z=0.50$ & $z=0.65$ \\
Drift rate 1 & $v_{l}=1.00$ & $v_{l}=1.00$ & $v_{l}=1.00$ & $v_{l}=1.00$ \\
Drift rate 2 & $v_{2}=-1.00$ & $v_{2}=-1.00$ & $v_{2}=-1.00$ & $v_{2}=-1.00$ \\
RT-constant & $t_{0}=0.400$ & $t_{0}=0.400$ & $t_{0}=0.400$ & $t_{0}=0.400$ \\
RT-bias & $d=0.000$ & $d=0.000$ & $d=0.050$ & $d=0.050$ \\
\hline
\end{tabular}

Note. A diffusion constant of $s=1$ was adopted. If other diffusion constants are of interest parameters $a, z$, and $v$ can be transformed by multiplication with the new value of $s$. 
Table 2. Means and SDs of inter-trial variability of starting point $\left(s_{z}\right)$, drift rate $\left(s_{v}\right)$, and RT constant $\left(s_{t 0}\right)$ as a function of parameter set and model restrictions (results from Study 1).

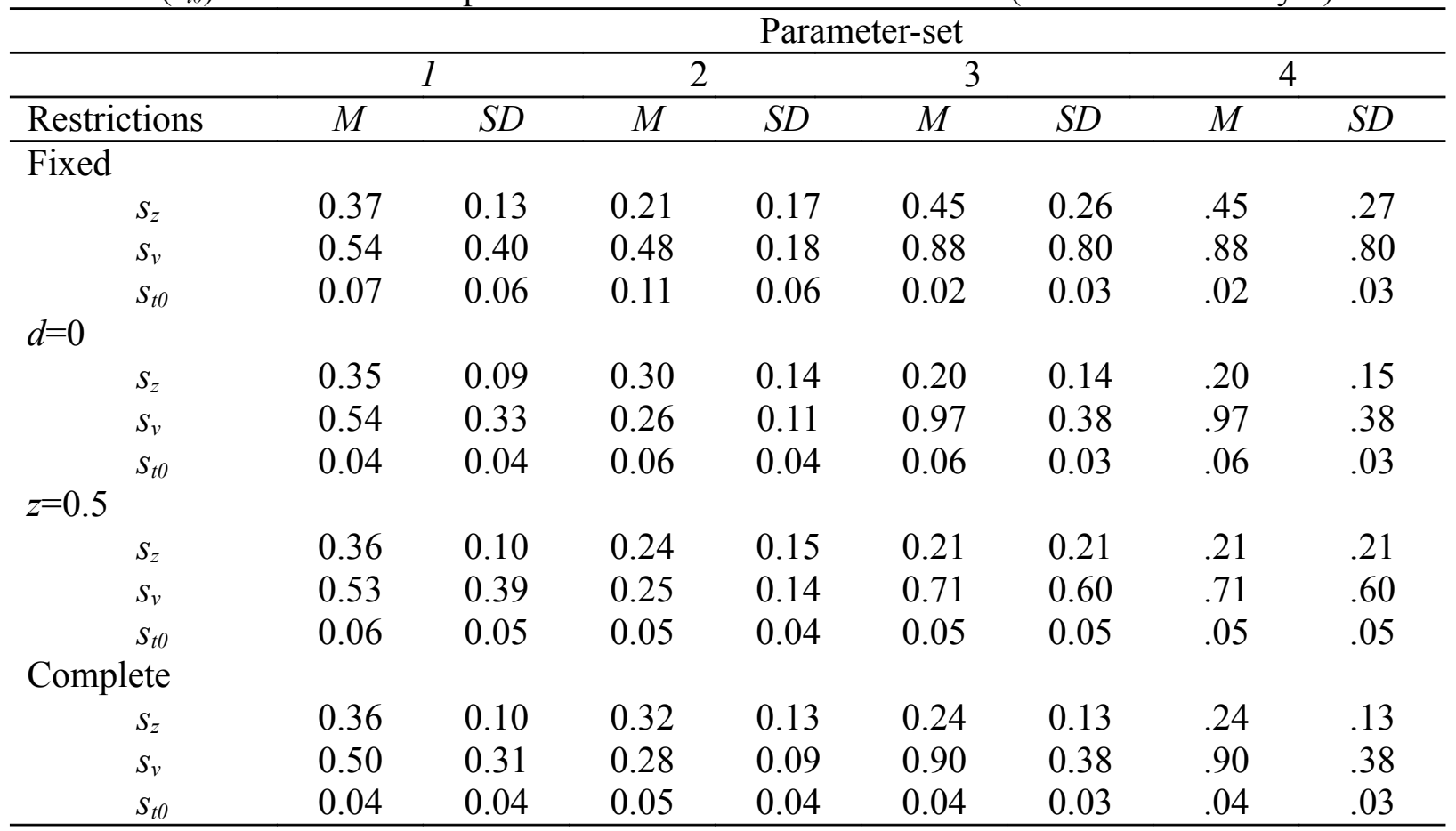

Note. Fixed: $d=0$ and $z=a / 2$; Complete: No restrictions of $d$ or $z$. 
Table 3. Model-fit indices of Study 1. The table shows the Kolmogorov-Smirnov-Statistic (KS), which is the maximum vertical distance between predicted and empiric RTdistributions.

\begin{tabular}{lcccccccc}
\hline & \multicolumn{8}{c}{ Parameter Set } \\
\hline Restrictions & $M$ & $S D$ & $M$ & $S D$ & $M$ & $S D$ & $M$ & $S D$ \\
\hline Fixed & .029 & .005 & $.056^{\mathrm{a}}$ & .008 & $.046^{\mathrm{a}}$ & .007 & $.078^{\mathrm{a}}$ & .007 \\
$d=0$ & .028 & .004 & .028 & .004 & $.034^{\mathrm{a}}$ & .005 & $.032^{\mathrm{a}}$ & .005 \\
$z=0.5$ & .026 & .004 & $.036^{\mathrm{a}}$ & .006 & .027 & .004 & $.037^{\mathrm{a}}$ & .007 \\
Complete & .027 & .003 & .027 & .003 & .029 & .004 & .030 & .005 \\
\hline
\end{tabular}

Note. Fixed: $d=0$ and $z=a / 2$; Complete: No restrictions of $d$ or $z$.

${ }^{a}$ Restrictions conflicted with the original values. 
Table 4. Ranges for parameter values for the simulated data sets from Study 2. The decision bias parameter was set to $z=0.5 a$, or $z=0.65 a$, respectively. The response-execution bias parameter was set to $d=0.05$ or $d=0.00$ respectively.

\begin{tabular}{crr}
\hline Parameter & Minimum & Maximum \\
\hline$a$ & 0.80 & 1.30 \\
$v_{l}$ & 1.00 & 4.00 \\
$v_{2}$ & -4.00 & -1.00 \\
$t_{0}$ & 0.20 & 0.40 \\
$s_{z}$ & 0.00 & $0.25 a$ \\
$s_{v}$ & 0.00 & 1.00 \\
$s_{t 0}$ & 0.00 & 0.10
\end{tabular}

Note. A diffusion constant of $s=1$ was adopted. If other diffusion constants are of interest parameters $a, z, v, s_{z}$, and $s_{v}$ can be transformed by multiplication with the new value of $s$. 
Figures

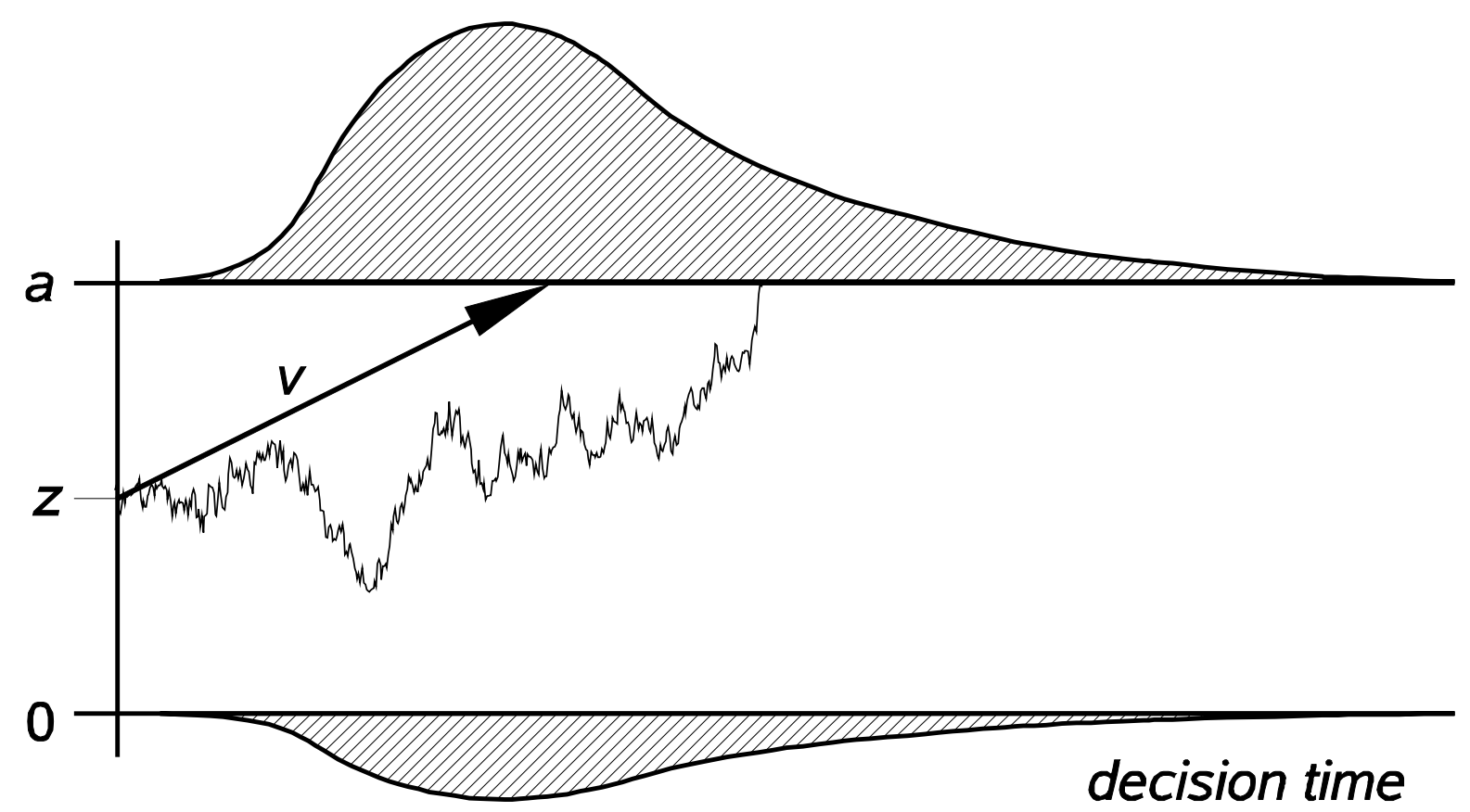

Figure 1. Ratcliff's Diffusion Model. 
A
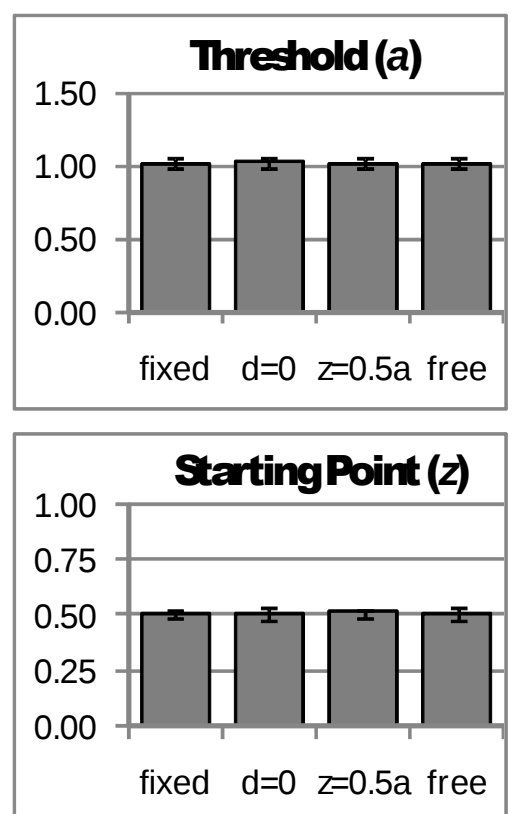

B
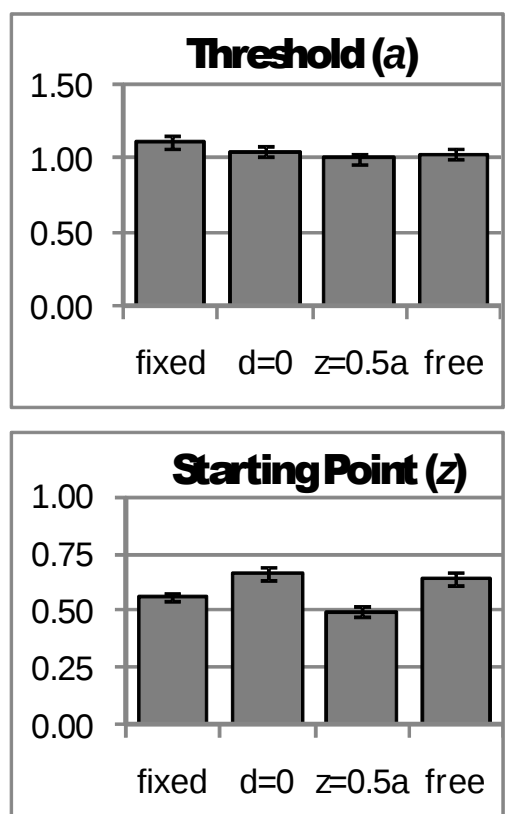
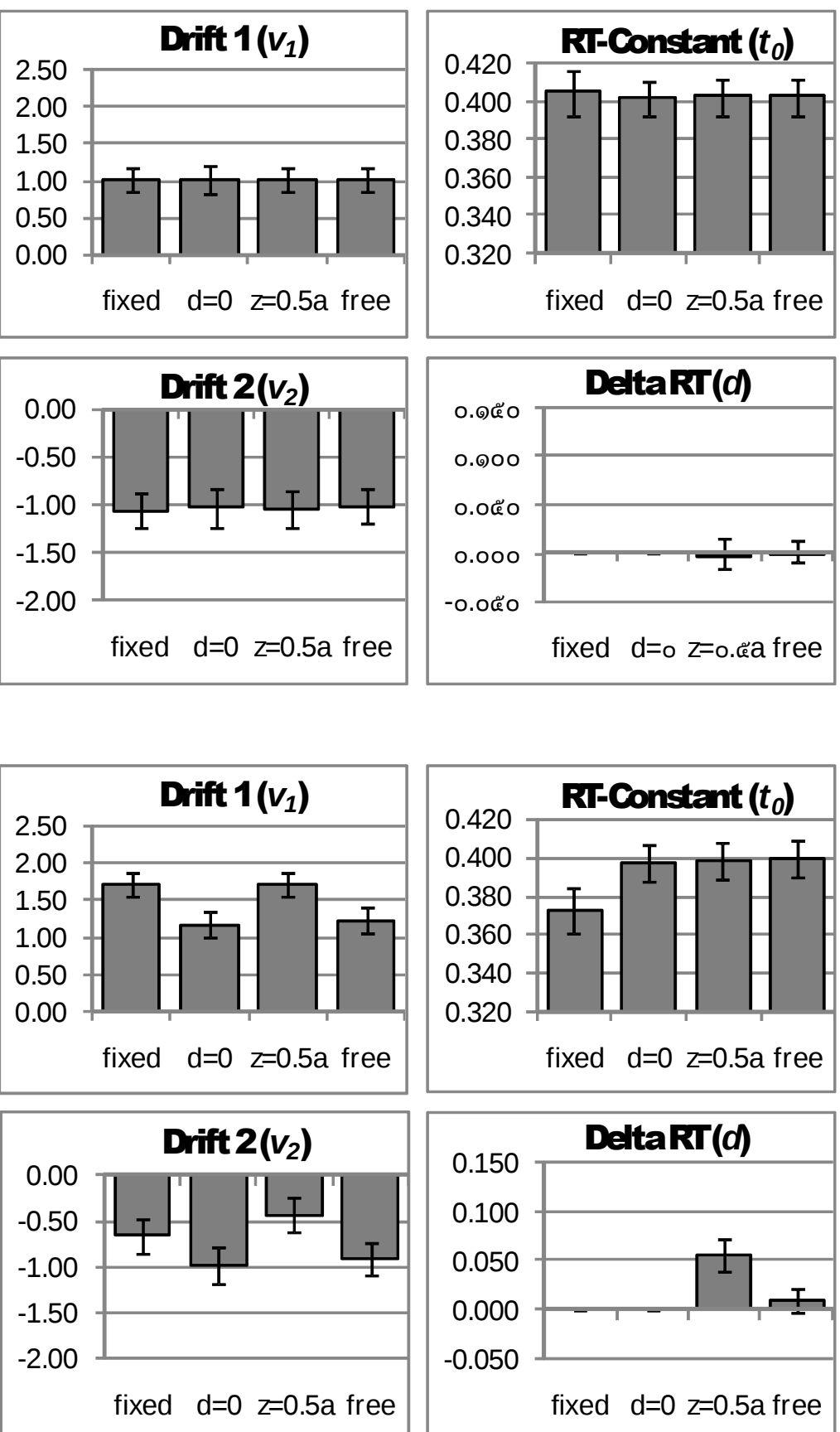

Figure 2. Re-estimated parameter values from Study 2. The top panel (A) depicts data from parameter set 1 (neither decision bias nor response-execution bias). The bottom panel (B) shows data from parameter set 2 (decision bias only). Original (correct) values are indicated by the slashed lines. Error bars indicate $+/-1 \mathrm{SD}$. 
C
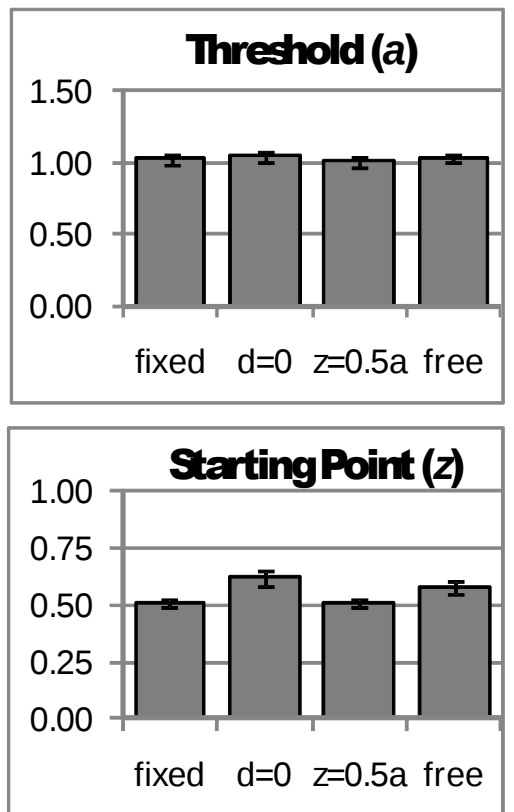

$\mathrm{D}$
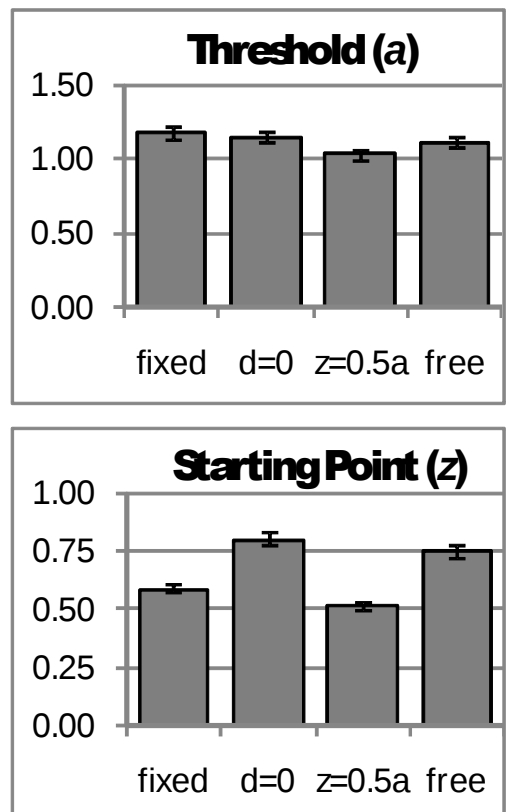
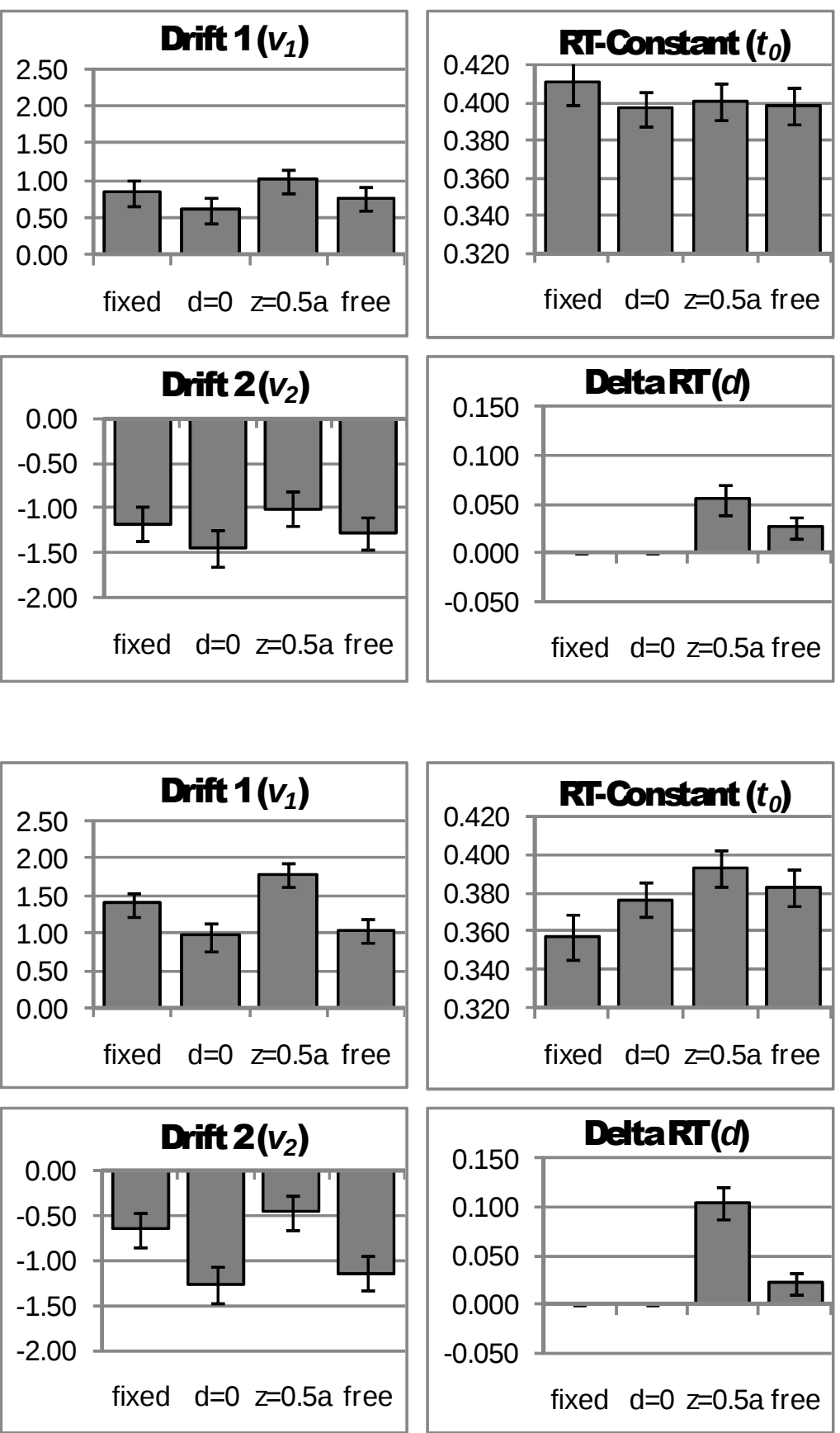

Figure 3. Re-estimated parameter values from Study 2. The top panel (C) shows data from parameter set 3 (response-execution bias only). The bottom panel (D) shows data from parameter set 4 (decision bias and response-execution bias). Original (correct) values are indicated by the slashed lines. Error bars indicate +/- $1 \mathrm{SD}$. 


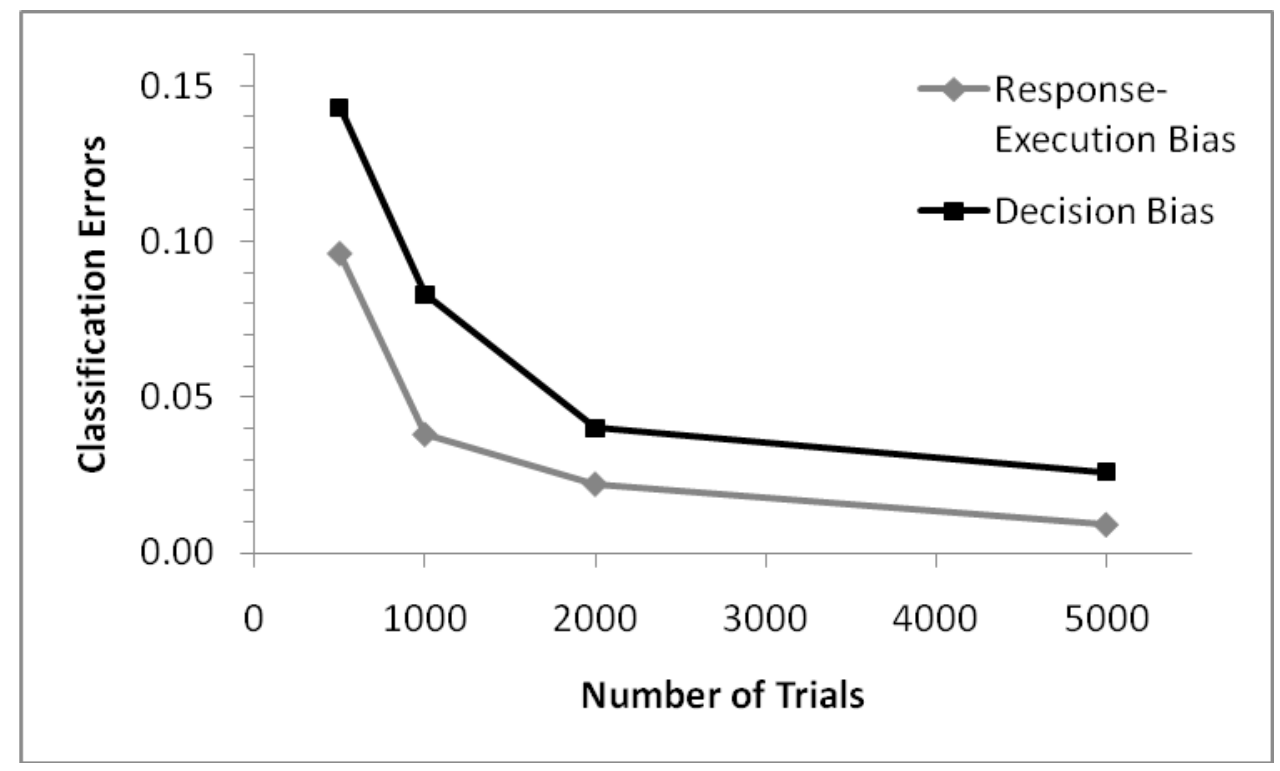

Figure 4. Percentage of data sets for which the estimation procedure with "wrong" restriction (i.e., incompatible with the original parameter set) resulted in a better fit than the models with "correct" restrictions (Study 2). The grey line represents data sets simulated from the response-execution bias model; the black line shows results from the decision-bias model. 
Response-Execution Bias Model
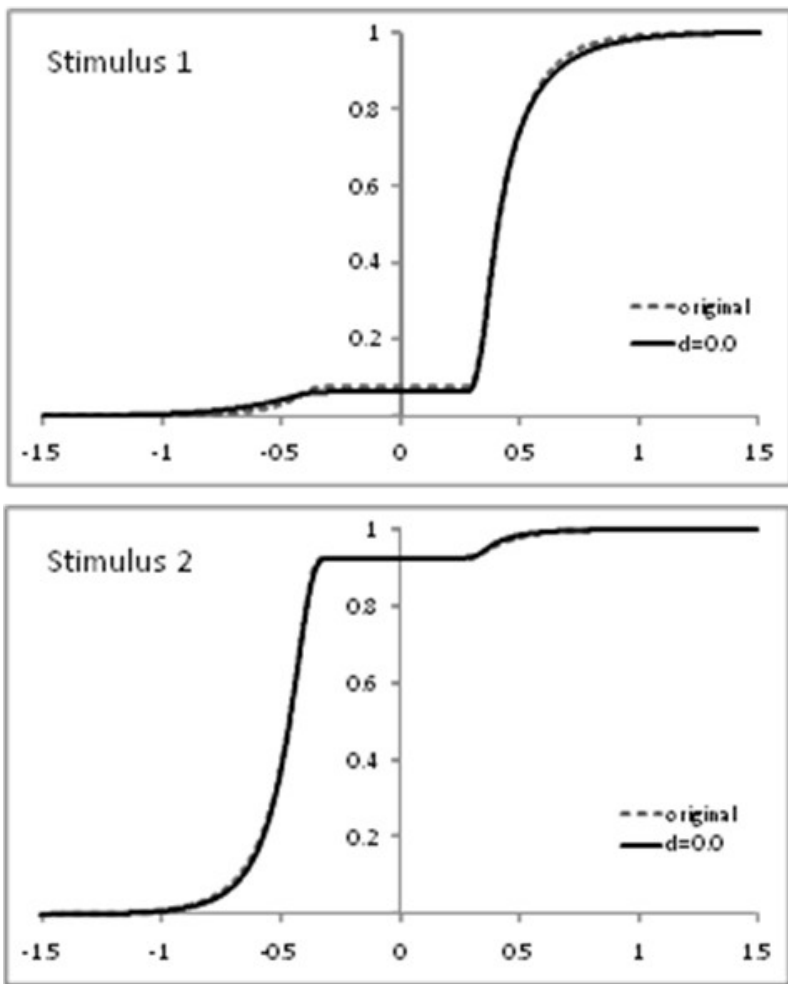

Decision-Bias Model
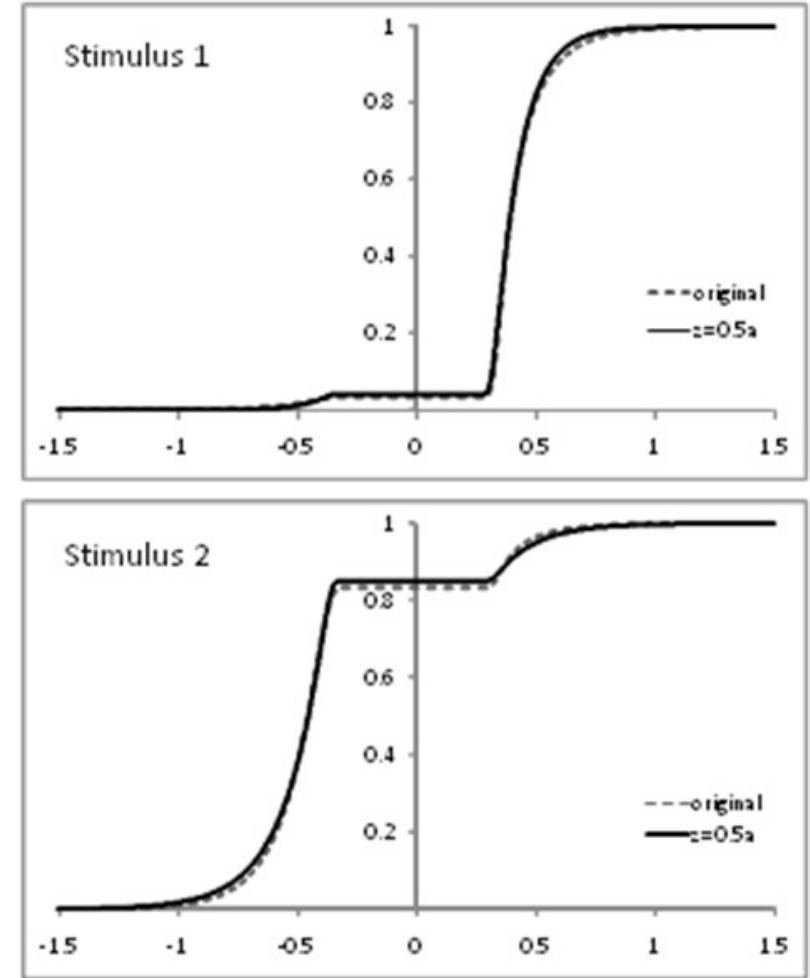

Figure 5. Model Mimicry: Combined Cumulative Distribution Functions (cCdFs) for the response-bias model (left) and for the decision-bias model (right). Original cCdFs are demonstrated by the dotted lines. The solid black lines give the cCdFs from parameters estimated with incorrect restrictions, that is, response-bias data is mimicked with a decisionbias model and vice versa. 